\title{
Fewer Layers, Faster Curing
}

Polyaspartic coatings cure very quickly and fewer layers of paint are often required. The development of a new hardener has increased the possibilities for using polyaspartic coatings on floors.

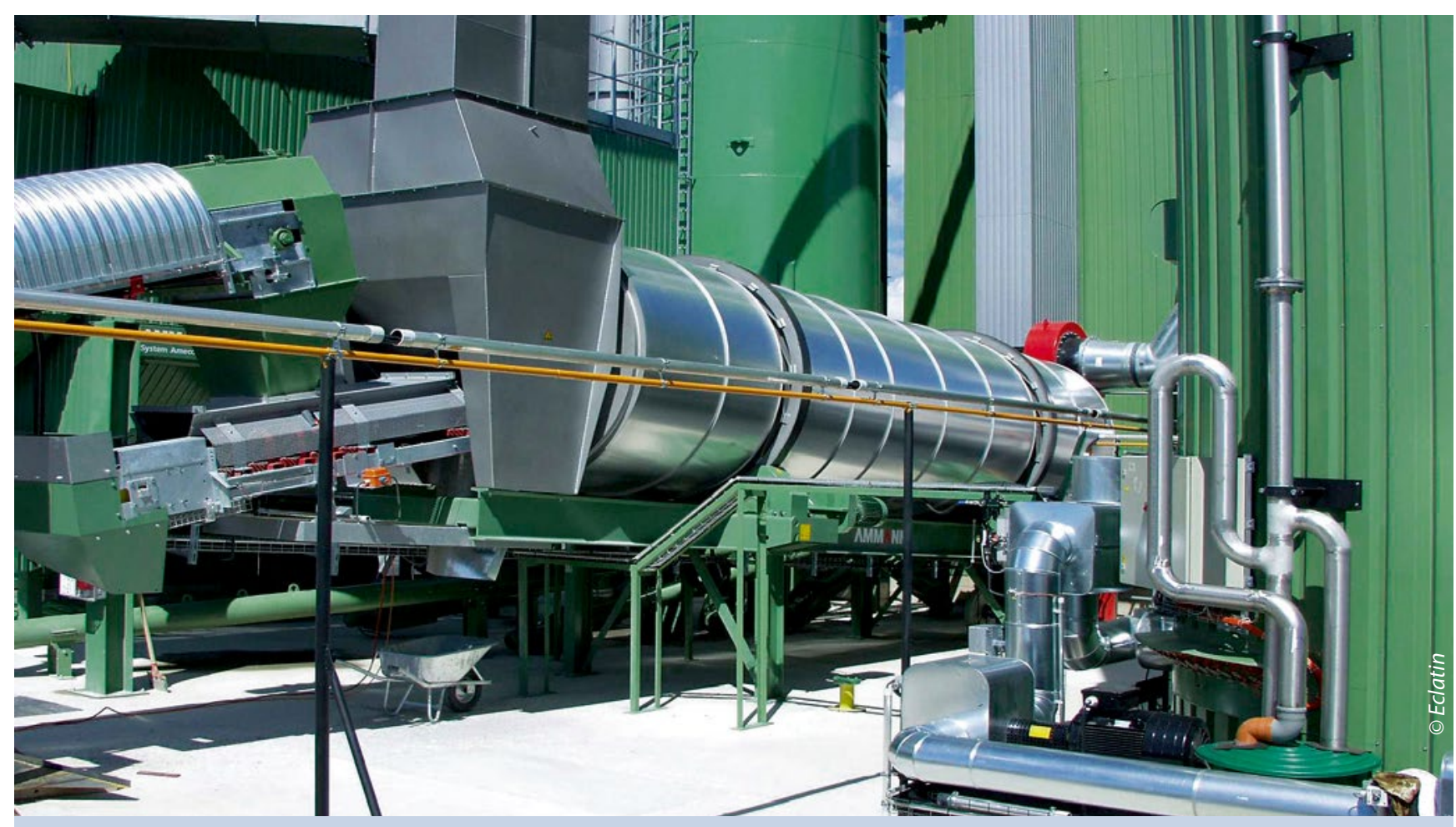

In Langenthal in Switzerland, the Ammann Group manufactures asphalt mixing machines, which are coated with polyaspartic systems. The paint is supplied by Eclatin AG, a company also based in Switzerland.

Dolyaspartic systems used for corrosion protection and as floor coatings require fewer layers and cure more rapidly than conventional solutions. The curing time can be reduced from between 18 and 24 hours to between only six and eight hours. This results in a two- to threefold increase in productivity. In addition, only a primer and base coat are needed, but the overall coating thickness remains unchanged, which ensures excellent corrosion protection.

\section{New hardener for flexible floor} coatings

For 15 years, polyaspartic coatings based on Bayer raw materials have proved their usefulness in protecting wind turbines, bridges, industrial plants and agricultural and construction machinery against corrosion, and have also functioned as floor coatings. Leading coating manufacturers include the systems in their product portfolios.

There is still some work to be done in terms of the application and the costeffectiveness when it comes to the use of this technology for floor coatings, however. This is why Bayer MaterialScience has developed Desmodur E 2863 $\mathrm{XP}$, a new hardener with very low viscosity. It can be used to produce flexible, solvent-free floor coatings which are suitable for outdoor use and for interior applications, such as odourless comfort flooring and sports flooring.

As a result of the rapid curing process, several layers of these polyaspartic coatings can be applied in a single day using standard equipment. Their pot life has been extended when compared with previous polyaspartic systems, which reduces the time pressure during the application process. The coatings can easily be applied at room temperature and the surface can be walked on after just a few hours. This keeps both downtime and costs to a minimum.

Bayer MaterialScience will continue developing its polyaspartic products, which will in future be sold under the new Pasquick umbrella brand. I

\section{Contact:}

Dr. Frank Rothbarth, Bayer MaterialScience, Leverkusen, Germany, Tel. +492143025363 ,

frank.rothbarth@bayer.com,

www.bayermaterialscience.com 